\title{
The effects of Kv1.3 and IKCa1 potassium channel inhibition on calcium influx of human peripheral T lymphocytes in rheumatoid arthritis
}

\author{
Gergely Toldi ${ }^{\mathrm{a}, *}$, Anna Bajnok ${ }^{\mathrm{a}}$, Diána Dobi ${ }^{\mathrm{b}}$, Ambrus Kaposi ${ }^{\mathrm{a}}$, László Kovács ${ }^{\mathrm{b}}$, Barna Vásárhelyi ${ }^{\mathrm{c}}$, \\ Attila Balog ${ }^{\mathrm{b}}$ \\ a First Department of Pediatrics, Semmelweis University, Budapest, Bókay u. 53-54, H-1083, Hungary \\ b Department of Rheumatology, Albert Szent-Györgyi Health Center, University of Szeged, Szeged, Kálvária sgt. 57, H-6027, Hungary \\ c Department of Laboratory Medicine, Semmelweis University, Budapest, Bókay u. 53-54, H-1083, Hungary
}

\section{A R T I C L E I N F O}

\section{Article history:}

Received 7 January 2012

Received in revised form 12 March 2012

Accepted 16 May 2012

\section{Keywords:}

Calcium influx

IKCa1

Kv1.3

Lymphocyte activation

Rheumatoid arthritis

\begin{abstract}
A B S T R A C T
Objective: The transient increase of the cytoplasmic free calcium level plays a key role in the process of lymphocyte activation. Kv1.3 and IKCa1 potassium channels are important regulators of the maintenance of calcium influx during lymphocyte activation and present a possible target for selective immunomodulation.

Design: Case-control study.

Subjects and methods: We took peripheral blood samples from 10 healthy individuals and 9 recently diagnosed rheumatoid arthritis (RA) patients receiving no anti-rheumatic treatment. We evaluated calcium influx kinetics following activation in CD4, Th1, Th2 and CD8 cells applying a novel flow cytometry approach. We also assessed the sensitivity of the above subsets to specific inhibition of the Kv1.3 and IKCa1 potassium channels.

Results: The peak of calcium influx in lymphocytes isolated from RA patients is reached more rapidly, indicating that they respond more quickly to stimulation compared to controls. In healthy individuals, the inhibition of the IKCa1 channel decreased calcium influx in Th2 and CD4 cells to a lower extent than in Th1 and CD8 cells. On the contrary, the inhibition of Kv1.3 channels resulted in a larger decrease of calcium entry in Th2 and CD4 than in Th1 and CD8 cells. No difference was detected between Th1 and Th2 or CD4 and CD8 cells in the sensitivity to IKCa1 channel inhibition among lymphocytes of RA patients. However, specific inhibition of the Kv1.3 channel acts differentially on calcium influx kinetics in RA lymphocyte subsets. Th2 and particularly CD8 cells are inhibited more dominantly than Th1 and CD4 cells.

Conclusion: The inhibition of Kv1.3 channels does not seem to be specific enough in peripheral RA lymphocytes, since anti-inflammatory $\mathrm{Th} 2$ cells are also affected to a noteworthy extent.
\end{abstract}

(c) 2012 Elsevier GmbH. All rights reserved.

\section{Introduction}

The short-term activation of peripheral blood and synovial fluid T lymphocytes, especially that of autoreactive T cells plays a crucial role in initiating and maintaining the chronic inflammation in the joints of patients suffering from rheumatoid arthritis (RA). These cells regulate the inflammatory process resulting in the destruction of the articular cartilage and also play a role in extra-articular

Abbreviations: AUC, area under the curve; $\left[\mathrm{Ca}^{2+}\right]_{\text {cyt }}$, cytoplasmic free calcium level; CRAC, calcium release activated calcium; Max, maximum value; MGTX, margatoxin; PBMC, peripheral blood mononuclear cell; PHA, phytohemagglutinin; RA, rheumatoid arthritis; TCM, central memory T cell; TEM, effector memory T cell; $t_{\max }$, time to reach maximum value; TRAM, triarylmethane.

* Corresponding author. Tel.: +36 20 4367181; fax: +3613138212.

E-mail address: toldigergely@yahoo.com (G. Toldi). damage. The transient increase of the cytoplasmic free calcium level $\left(\left[\mathrm{Ca}^{2+}\right]_{\text {cyt }}\right)$ is a cornerstone in the process of lymphocyte activation and pathological functioning of lymphocytes in RA. The engagement of the TCR/CD3 complex upon antigen presentation leads to calcium release from intracellular stores that is followed by further calcium entry from the extracellular space through the store-operated calcium release activated calcium (CRAC) channels. The influx of calcium is maintained by the function of potassium channels that conserve the electrochemical potential gradient via the efflux of potassium from the cytoplasm. There are two major types of potassium channels in T cells: the voltage-gated Kv1.3 and the calcium-activated IKCa1 channels. Kv1.3 channels are activated upon the depolarization of the cell and sense alterations of the membrane potential. In contrast, IKCa 1 channels are activated by the rise of $\left[\mathrm{Ca}^{2+}\right]_{\text {cyt }}$ above $100 \mathrm{nM}$ (Hu et al. 2007; Panyi 2005).

In spite of the increasing number of modern targeted therapeutic strategies (such as anti-TNF alpha or anti-IL-6 agents), there is 
limited data available on their specific effect on disease-causing autoreactive lymphocytes. Therefore, novel immunomodulators with improved specificity for autoreactive $\mathrm{T}$ cells are needed. Recent reports raised the notion that the inhibition of lymphocyte potassium channels, especially that of the Kv1.3 channel would be an optimal solution for specific immunosuppression in autoimmune disorders, including RA (Beeton et al. 2001, 2006; Wulff et al. 2003). The relationship between the calcium currents through CRAC channels and the efflux of potassium makes the proliferation and activation of lymphocytes sensitive to pharmacological modulation of Kv1.3 and IKCa1 channels, and provides an opportunity for targeted intervention. Experimentally, specific inhibition of these channels results in a diminished calcium influx in lymphocytes and a lower level of lymphocyte activation (Panyi et al. 2006).

Beeton et al. (2006) demonstrated that disease associated autoreactive $\mathrm{T}$ cells isolated from RA patients are principally CCR7-CD45RA- effector memory $T$ cells (TEM cells) with elevated Kv1.3 potassium channel expression, in contrast with naive and central memory T cells (TCM cells) that express low levels of Kv1.3 channels. Hence, Kv1.3 inhibitors preferentially suppress calcium signaling, cytokine production and proliferation of autoantigen-specific TEM cells while sparing other classes of $\mathrm{T}$ cells. Furthermore, Beeton and colleagues evaluated the therapeutic potential of Kv1.3 inhibitors in pristane-induced chronic arthritis, a rat model of RA. Animals treated with a specific blocker of the Kv1.3 channel had significantly fewer affected joints and showed significant improvement in radiological and histopathological findings (Beeton et al. 2006). Additionally, no clinical signs of toxicity were identified during the trial.

However, systemic treatment with lymphocyte potassium channel inhibitors would affect lymphocytes not only locally in the synovium, but also those in peripheral blood. Therefore, in this study we aimed to characterize the effects of lymphocyte potassium channel inhibition on short-term peripheral blood $\mathrm{T}$ lymphocyte activation in major lymphocyte subsets of patients recently diagnosed with RA. We employed a kinetic flow cytometry method to describe calcium influx characteristics of the CD4, Th1, Th2 and CD8 subsets and its sensitivity to the inhibition of Kv1.3 and IKCa1 lymphocyte potassium channels.

\section{Materials and methods}

\section{Patients}

We enrolled 10 healthy individuals and 9 recently diagnosed RA patients (early RA patients), who had not received antirheumatic treatment, including non-steroidal anti-inflammatory drugs (NSAIDs), disease modifying anti-rheumatic drugs (DMARDs) and glucocorticoids until the time of blood sampling. Clinical parameters of study participants are summarized in Table 1. RA patients were diagnosed according to the latest ACR/EULAR criteria (Aletaha et al. 2010). Healthy controls had a negative history of rheumatic symptoms and negative status upon detailed physical and laboratory examination. No co-morbidities were detected in patients and controls that could have influenced our investigation, nor did they take any medication that could have interfered with the measurements. Written informed consent was obtained from all subjects, and our study was reviewed and approved by an independent ethical committee of the university. Laboratory studies and interpretations were performed on coded samples lacking personal and diagnostic identifiers. The study was adhered to the tenets of the most recent revision of the Declaration of Helsinki.
Table 1

Clinical characteristics of study participants.

\begin{tabular}{lll}
\hline Characteristics & $\begin{array}{l}\text { Healthy individuals } \\
n=10\end{array}$ & $\begin{array}{l}\text { RA patients } \\
n=9\end{array}$ \\
\hline Age (years) & $51[46-57]$ & $58[44-61]$ \\
Gender (male/female) & $4 / 6$ & $4 / 5$ \\
RA duration (months) & - & $3[2-4.5]$ \\
RA criteria score & - & $8[8-10]$ \\
No. of swollen joints & - & $8[6.5-13]$ \\
No. of tender joints & - & $14[11-18]$ \\
RF $(\mathrm{U} / \mathrm{mL})$ & BLD & $96[62-292]$ \\
Anti-MCV $(\mathrm{U} / \mathrm{mL})$ & BLD & $469[29-963]$ \\
CRP $(\mathrm{mg} / \mathrm{L})$ & $2.7[0.0-3.9]$ & $30.6^{\mathrm{a}}[16.1-63.0]$ \\
We $(\mathrm{mm} / \mathrm{h})$ & $10[7-14]$ & $50^{\mathrm{a}}[44-74]$ \\
\hline
\end{tabular}

Data are expressed as median [interquartile range] for continuous variables and as number for categorical variables.

${ }^{a} p<0.05$ vs. healthy individuals.

b According to the latest ACR/EULAR guidelines [7].

$\mathrm{BLD}$ - below the level of detection, $\mathrm{CRP}-\mathrm{C}$ reactive protein, $\mathrm{MCV}$ - mutated citrullinated vimentin, RA - rheumatoid arthritis, RF - rheumatoid factor, We - erythrocyte sedimentation rate.

\section{Fluorescent staining}

Our measurements were carried out as described earlier (Toldi et al. 2011). Briefly, peripheral blood mononuclear cells (PBMCs) were isolated by a standard density gradient centrifugation from $9 \mathrm{~mL}$ of freshly drawn peripheral venous blood and afterwards kept in a modified RPMI medium (calcium concentration: $2 \mathrm{mM}$ ) throughout the following steps of the procedure. PBMCs were then incubated with the following conjugated anti-human monoclonal antibodies in order to differentiate $\mathrm{T}$ lymphocyte subsets (Fig. 1): anti-CD4 PE-Cy7, anti-CD8 APC-Cy7, anti-CXCR3 APC (for the determination of Th1 cells) and anti-CCR4 PE (for the determination of Th2 cells) (all from PharMingen, San Diego, CA, USA), as well as anti-Kv1.3 channel FITC (Sigma-Aldrich, St. Louis, MO, USA), according to the manufacturers' instructions. For monitoring $\left[\mathrm{Ca}^{2+}\right]_{\mathrm{cyt}}$, PBMCs were loaded with calcium sensitive Fluo-3 and Fura-Red dyes according to the manufacturer's recommendations (Invitrogen, Carlsbad, CA, USA).

\section{Flow cytometry}

PBMCs were equally distributed into three vials. The first vial was used as control. The second vial was treated with margatoxin (MGTX, $60 \mathrm{nM}$ ), a selective blocker of the Kv1.3 channel. The third vial was treated with a triarylmethane compound (TRAM, $60 \mathrm{nM}$ ), a specific inhibitor of the IKCa1 channel. PBMCs were activated by the addition of $20 \mu \mathrm{g}$ phytohemagglutinin (PHA) and the measurements were initiated directly afterwards on a BD FACSAria flow cytometer. Cell fluorescence data were measured and recorded for $10 \mathrm{~min}$ in a kinetic manner.

\section{Data evaluation}

Recordings were evaluated with our specific software (FacsKin), based on the calculation of a double-logistic function for each recording (Kaposi et al. 2008; Toldi et al. 2011). This function is used to characterize measurements that have an increasing and a decreasing intensity as time passes. The software also calculated parameter values describing each function, such as the Area Under the Curve (AUC), Maximum (Max), time to reach maximum $\left(t_{\max }\right)$, and Slope values. AUC values correspond to the sum of $\left[\mathrm{Ca}^{2+}\right]_{\mathrm{cyt}}$ increase, which further corresponds to the level of lymphocyte activation (Panyi 2005). Max values represent the peak value of the calcium influx curve upon lymphocyte activation. $t_{\max }$ values describe how soon the peak value of the calcium influx curve is reached. The Slope value reflects how rapidly the peak of calcium 


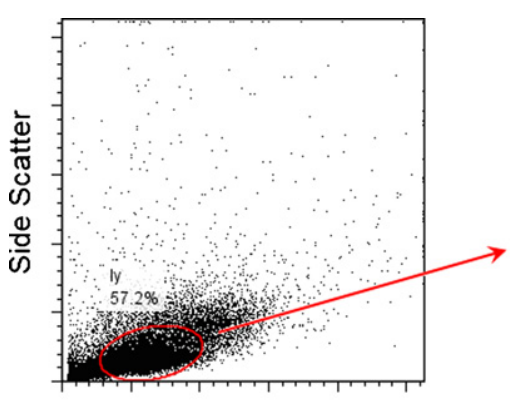

Forward Scatter
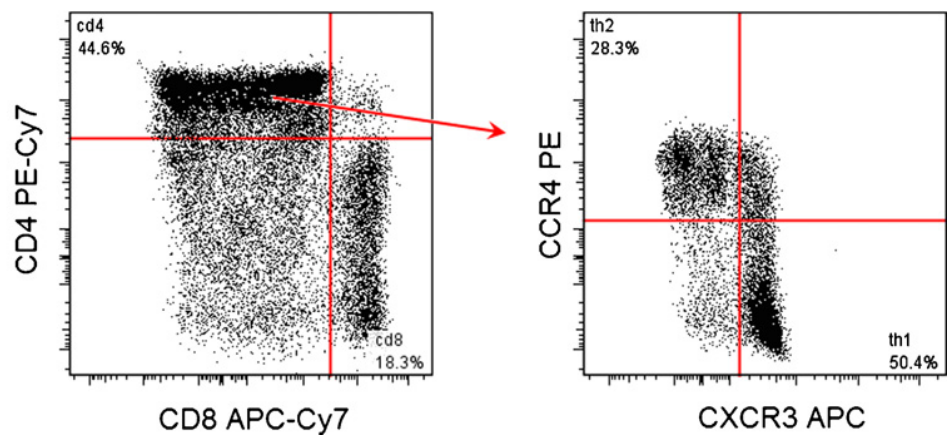

Fig. 1. Gating strategy used for differentiation between CD4, CD8, Th1 (CD4+ CXCR3+) and Th2 (CD4+ CCR4+) cells. ly - lymphocytes.

influx is reached. A detailed description of the evaluation process can be found at www.facskin.com.

\section{Statistics}

Data are expressed as median and interquartile range. Comparisons between two sample groups were made with the Mann-Whitney test. For comparisons between paired values in the same group Wilcoxon tests were applied. $p$ values less than 0.05 were considered significant. Statistics were calculated using the $\mathrm{R}$ software.

\section{Results}

\section{Clinical data}

As seen in Table 1, the age and gender distribution of participants were similar in both study groups. Inflammatory parameters (erythrocyte sedimentation rate and $C$ reactive protein values) were higher in RA patients than in healthy controls.

\section{The frequency of the investigated lymphocyte subsets}

We determined the frequency of CD4, Th1, Th2 and CD8, cells in samples of both study groups (Table 2). CD4+ CXCR3+ cells were regarded as Th1 lymphocytes, while CD4+ CCR4+ cells were regarded as the Th2 subset. While no significant difference was detected regarding individual cell subsets among the study groups, the ratio of Th1 and Th2 cells was significantly lower in the RA group in comparison with healthy individuals.

\section{Calcium influx kinetics during lymphocyte activation}

The calculated parameter values (AUC, Max, $t_{\max }$ and Slope) were compared following lymphocyte activation with PHA between healthy subjects and RA patients. The parameters are

\section{Table 2}

Prevalence of lymphocyte subsets in the overall lymphocyte population gated according to Forward Scatter Characteristics (FSC) and Side Scatter Characteristics (SSC) in 10 healthy individuals and 9 recently diagnosed RA patients.

\begin{tabular}{lll}
\hline Subset & Healthy & RA \\
\hline CD4+/ly & $38.6[33.0-53.1]$ & $41.8[34.8-48.1]$ \\
CXCR3+/CD4 & $43.5[35.5-51.0]$ & $40.2[29.7-46.7]$ \\
CCR4+/CD4 & $17.7[11.4-22.8]$ & $24.0[17.0-30.4]$ \\
CD4+ CXCR3+/ & $3.03[1.82-3.82]$ & $1.50^{\mathrm{a}}[1.10-2.80]$ \\
$\quad$ CD4+ CCR4+ ratio & $15.7[8.80-23.6]$ & $9.40[7.64-18.5]$ \\
CD8+/ly & 15.7 & 9 \\
\hline
\end{tabular}

Data are expressed as median [interquartile range]. CD4+ CXCR3+- Th1 subset, CD4+ CCR4+ - Th2 subset, ly - overall lymphocyte population, RA - rheumatoid arthritis. a $p<0.05$ vs. healthy individuals shown in Table 3 and Fig. 2. Slope values showed no alteration between the study groups in the investigated lymphocyte subsets, and therefore are not included in Table 3. Within the CD4 population, AUC and $t_{\max }$ values of calcium influx were decreased in RA patients compared with healthy controls. When focusing on the Th1 subset, the decrease was present in the AUC, Max and $t_{\max }$ values, while in Th2 lymphocytes solely the $t_{\max }$ value was lower. We found no difference in the parameters of calcium influx in the CD8 subset.

\section{The effects of potassium channel inhibitors on lymphocyte calcium influx}

We evaluated the effect of specific inhibitors of the Kv1.3 and IKCa1 channels (MGTX and TRAM, respectively) on parameter values of calcium influx in both study groups. Results are shown in Table 3 and Fig. 2. Slope values showed no alteration in the investigated lymphocyte subsets of the study groups upon inhibitor treatment. MGTX and TRAM treatment decreased AUC values of all investigated lymphocyte subsets in samples of both study groups. The Max value was decreased in all investigated lymphocyte subsets of healthy individuals and RA patients by MGTX. TRAM induced a decrease in the Max value of Th1, Th2 and CD8 cells isolated from healthy individuals, and in all investigated lymphocyte subsets of RA patients. The $t_{\max }$ value was only decreased by TRAM in the CD8 subset of RA patients. The extent of the decrease in parameter values was characteristic for the investigated study group and the inhibited potassium channel in each subset, as detailed in "Discussion".

\section{Kv1.3 channel expression in the investigated lymphocyte subsets}

We evaluated the median fluorescence of the antibody against Kv1.3 channels in lymphocytes of both study groups. Median fluorescence was higher in RA patients compared with healthy individuals in the CD4+ (54 [39-64] vs. 37 [22-43] arbitrary units, $p<0.05$ (median [interquartile range])) and the CD4+ CXCR3+ (53 [36-69] vs. 14 [4-25] arbitrary units, $p<0.05$ (median [interquartile range])) subsets. No significant alterations were found in the median fluorescence in the CD4+ CCR4+ and CD8+ subsets.

\section{Basal cytoplasmic calcium levels in the investigated lymphocyte subsets}

We evaluated the ratio of the basal median fluorescence of calcium binding dyes in lymphocytes of both study groups. Basal median fluorescence was higher in RA patients compared with healthy individuals in the CD4+ $(11,605$ [5617-14,104] vs. 7858 [3922-10,743] arbitrary units, $p<0.05$ (median [interquartile range])), the CD4+ CCR4+ $(11,064$ [7188-14,631] vs. 5920 
Table 3

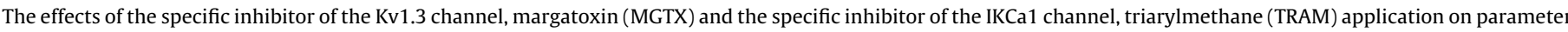

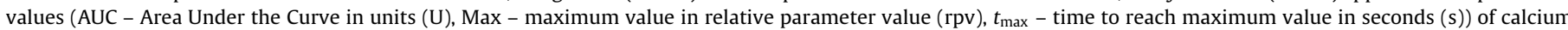
influx kinetics in peripheral lymphocytes obtained from 10 healthy individuals and 9 recently diagnosed RA patients.

\begin{tabular}{|c|c|c|c|c|c|c|c|}
\hline \multirow[t]{2}{*}{ Subset } & & \multicolumn{2}{|l|}{ No inhibitor } & \multicolumn{2}{|l|}{$\operatorname{MGTX}(60 \mathrm{nM})$} & \multicolumn{2}{|l|}{ TRAM (60 nM) } \\
\hline & & Healthy & RA & Healthy & RA & Healthy & RA \\
\hline \multirow{3}{*}{ CD4+ } & AUC (U) & $790[738-871]$ & $676^{\mathrm{a}}[665-787]$ & $89 \%^{\mathrm{b}}[83-93 \%]$ & $91 \%$ c [88-96\%] & $93 \%$ b [89-97\%] & $93 \%$ [90-96\%] \\
\hline & Max (rpv) & $1.383[1.228-1.647]$ & $1.297[1.183-1.390]$ & $94 \%^{\mathrm{b}}$ [83-99\%] & $89 \%{ }^{\mathrm{c}}[80-90 \%]$ & $95 \%[90-100 \%]$ & $90 \%$ c $[88-99 \%]$ \\
\hline & $t_{\max }(\mathrm{s})$ & $416[261-563]$ & $174^{\mathrm{a}}[140-309]$ & $90 \%$ [39-184\%] & $117 \%$ [111-171\%] & $99 \%[89-132 \%]$ & $98 \%[80-127 \%]$ \\
\hline \multirow{3}{*}{$\begin{array}{l}\text { CD4+ } \\
\text { CXCR3+ }\end{array}$} & $\operatorname{AUC}(\mathrm{U})$ & 897 [805-1006] & $761^{a}$ [719-797] & $91 \% \mathrm{~b}$ [84-94\%] & $93 \% c[85-93 \%]$ & $86 \%$ b [85-93\%] & $92 \%$ [ $[88-94 \%]$ \\
\hline & Max (rpv) & $1.660[1.466-1.846]$ & $1.387^{\mathrm{a}}[1.279-1.439]$ & $87 \%$ b [82-97\%] & $90 \%{ }^{\mathrm{c}}[84-92 \%]$ & $89 \%$ b [85-94\%] & $91 \% \mathrm{c}[86-93 \%]$ \\
\hline & $t_{\max }(\mathrm{s})$ & $396[227-515]$ & $289^{a}[168-358]$ & $118 \%$ [66-162\%] & $108 \%$ [26-196\%] & $116 \%$ [92-221\%] & $82 \%$ [58-117\%] \\
\hline \multirow{3}{*}{$\begin{array}{l}\text { CD4+ } \\
\text { CCR4+ }\end{array}$} & $\operatorname{AUC}(\mathrm{U})$ & $790[751-857]$ & 735 [697-777] & $86 \%$ b [84-93\%] & $89 \%$ c [85-94\%] & $92 \%$ b $[88-94 \%]$ & $91 \%$ [ $[88-96 \%]$ \\
\hline & $\operatorname{Max}(\mathrm{rpv})$ & 1.459 [1.249-1.574] & 1.357 [1.277-1.462] & $88 \%^{\mathrm{b}}$ [79-90\%] & $84 \% \mathrm{c}[79-91 \%]$ & $88 \%^{\mathrm{b}}[86-98 \%]$ & $91 \%$ [ $[82-94 \%]$ \\
\hline & $t_{\max }(\mathrm{s})$ & 365 [214-537] & $192^{\mathrm{a}}[151-273]$ & $110 \%$ [64-174\%] & $104 \%$ [99-132\%] & $125 \%$ [97-164\%] & $91 \%[88-113 \%]$ \\
\hline \multirow{3}{*}{ CD8+ } & AUC $(U)$ & $831[742-950]$ & 821 [734-905] & $96 \%{ }^{\mathrm{b}}[87-98 \%]$ & $85 \%$ c [78-89\%] & $89 \%$ b [83-91\%] & $91 \%$ [ $[85-93 \%]$ \\
\hline & $\operatorname{Max}(\mathrm{rpv})$ & $1.462[1.260-1.918]$ & $1.545[1.282-1.620]$ & $92 \%$ b [88-97\%] & $86 \%$ c [78-88\%] & $85 \%$ b [77-89\%] & $89 \%{ }^{\mathrm{c}}[87-93 \%]$ \\
\hline & $t_{\max }(\mathrm{s})$ & $326[253-478]$ & $368[212-501]$ & $97 \%$ [68-133\%] & $110 \%[37-126 \%]$ & $108 \%$ [68-163\%] & $59 \%$ c [49-92\%] \\
\hline
\end{tabular}

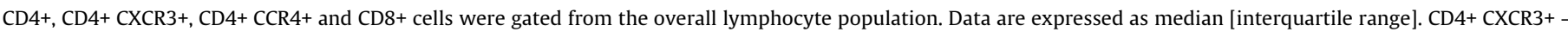
Th1 subset, CD4+ CCR4+ - Th2 subset, RA - rheumatoid arthritis.

a Lymphocytes isolated from RA patients were compared with lymphocytes isolated from healthy individuals within samples with no inhibitor application, $p<0.05$.

b MGTX and TRAM treated samples were compared with samples with no inhibitor application within lymphocytes isolated from healthy individuals, $p<0.05$.

${ }^{c}$ MGTX and TRAM treated samples were compared with samples with no inhibitor application within lymphocytes isolated from RA patients, $p<0.05$.

[3123-9863] arbitrary units, $p<0.05$ (median [interquartile range])) and the CD8+(9486 [6072-14,922] vs. 6445 [3477-14,673] arbitrary units, $p<0.05$ (median [interquartile range])) subsets.

\section{Discussion}

In this study, we investigated T lymphocyte calcium influx kinetics following activation in peripheral blood of recently diagnosed
RA patients compared to healthy individuals. An advantage of our investigation is that all patients were enrolled very soon after the onset of disease (a few months after the presentation of the first symptoms). Therefore, they had not received any anti-rheumatic treatment until the time of blood sampling. It is important to note that they were diagnosed according to the latest ACR/EULAR guidelines (Aletaha et al. 2010) and all fulfilled the criteria of early definitive RA.

\section{CD4 cells}
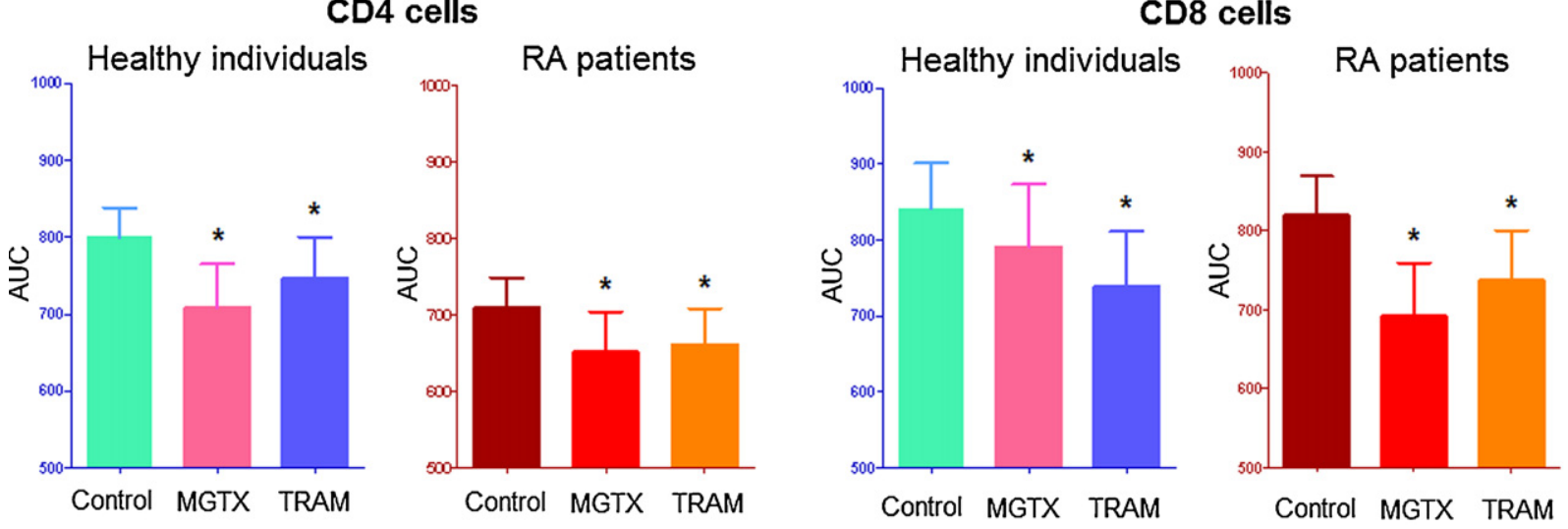

Th1 cells
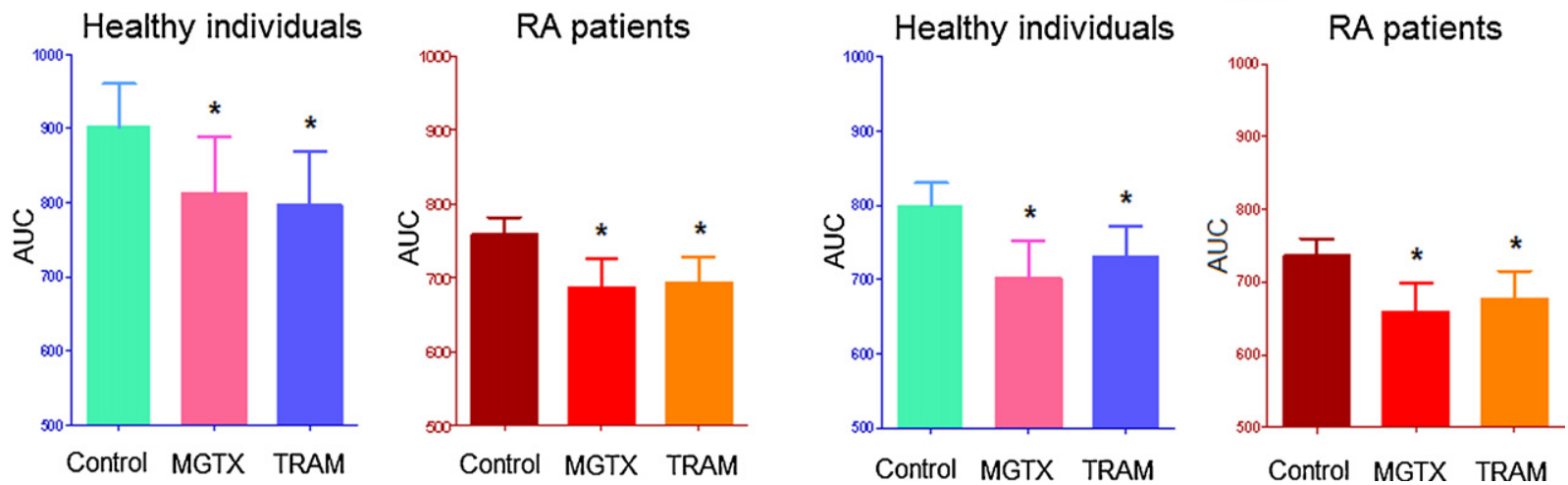

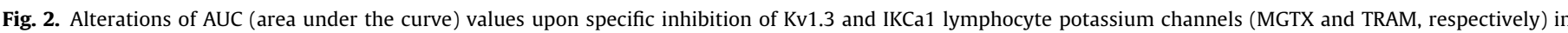
healthy individuals and rheumatoid arthritis (RA) patients. ${ }^{*} p<0.05$ vs. control, graph-median, whisker-interquartile range. 


\section{Th1/Th2 ratio in $R A$ patients and healthy subjects}

Based on the expression of cell surface chemokine receptors, the Th1/Th2 ratio was lower in RA patients than in healthy subjects. Although the difference of CD4+ CCR4+ (Th2) cell frequency did not reach the level of statistical significance in our study, we observed a tendency of higher frequency of this subset in RA patients in comparison with healthy individuals in peripheral blood. The increased expression of CCR4 has been previously noted by other investigations in RA, ankylosing spondylitis, and systemic lupus erythematosus (Szalay et al. 2012; Yang et al. 2004). Furthermore, Yang et al. (2004) demonstrated that the frequency of CD4+ CCR4+ cells was closely correlated with the elevated serum level of IL-10 in these disorders. These findings indicate that Th2 responses might be enhanced in the above mentioned diseases. Although RA is regarded as a Th1 polarized disease based on the elevated presence of pro-inflammatory cytokines (Bucht et al. 1996; Dolhain et al. 1996; van der Graaff et al. 1999), Th2 responses might also be enhanced in peripheral blood, however, the immunoregulatory role of Th2 cells may be insufficient to control increased Th1 responses.

\section{Calcium influx kinetics in RA patients and healthy subjects}

In line with our previous study in another autoimmune disorder, multiple sclerosis, we found that lymphocytes isolated from RA patients respond more quickly to stimulation than those of healthy individuals (Toldi et al. 2011). This is represented by lower $t_{\max }$ values, i.e. calcium influx reaches its peak earlier upon activation compared to healthy controls (Table 3 ). This finding is especially relevant in the Th2 subset, also indicating that Th2 responses might be enhanced in RA to control the ongoing inflammation. The increased reactivity of RA lymphocytes has already been noted by previous investigations. Buntinx et al. demonstrated that RA T cells show a sustained elevation of $\left[\mathrm{Ca}^{2+}\right]_{\text {cyt }}$ compared with healthy controls when activated in the presence of interferon (IFN) gamma, along with a significantly augmented proliferation in response to suboptimal doses of PHA. Their results suggest that the IFN gammainduced calcium influx acts as a complementary mechanism in the activation of $\mathrm{T}$ cells from RA patients besides the conventional TCR/CD3-mediated pathway (Buntinx et al. 2002).

Basal $\left[\mathrm{Ca}^{2+}\right]_{\text {cyt }}$ levels in $\mathrm{RA}$ patients and healthy subjects

The findings of our study indicate that basal $\left[\mathrm{Ca}^{2+}\right]_{c y t}$ was elevated in RA compared to healthy individuals. Nagy et al. also demonstrated earlier that peripheral blood T lymphocytes of RA patients are characterized by an increase in intracellular nitric oxide (NO) production along with elevated basal $\left[\mathrm{Ca}^{2+}\right]_{\text {cyt }}$ levels (Nagy et al. 2008). An important outcome of their finding might be the effects of $\left[\mathrm{Ca}^{2+}\right]_{c y t}$ on gene expression, in particular that of inflammatory cytokine. Distinct characteristics of calcium influx kinetics have been demonstrated to differentially influence the production of pro- and anti-inflammatory cytokines (Dolmetsch et al. 1998; Verheugen and Vijverberg 1995).

\section{The effects of potassium channel inhibitors on lymphocyte calcium} influx

Our results revealed that the sensitivity of Th1 and Th2 cells to IKCa1 channel inhibition was different in lymphocytes isolated from healthy individuals. Treatment with TRAM, the specific inhibitor of the IKCa1 channel decreased calcium influx in Th2 cells to a lower extent than in Th1 cells. On the contrary to IKCa1, the inhibition of Kv1.3 channels resulted in a larger decrease of calcium entry in Th2 than in Th1 cells. This is in line with previous

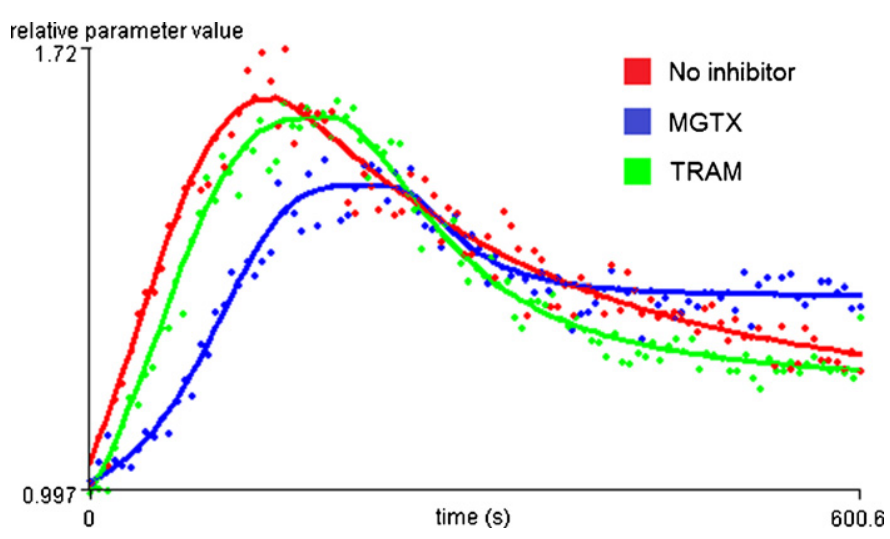

Fig. 3. The effects of Kv1.3 and IKCa1 potassium channel inhibitors on calcium influx kinetics in CD8 lymphocytes of recently diagnosed RA patients (data of a representative sample). Kinetic flow cytometry measurements indicate that compared to lymphocytes with no inhibitor treatment, the application of margatoxin (MGTX), the specific blocker of the Kv1.3 channel resulted in a greater decrease of calcium entry (represented by relative parameter value) than that of triarylmethane (TRAM), the specific inhibitor of the IKCa1 channel upon activation of samples with phytohemagglutinin.

findings of ours and others (Fanger et al. 2000; Toldi et al. 2012). In healthy individuals, CD4 cells were more sensitive to the inhibition of Kv1.3 channels than the CD8 subset, responding with a higher level of decrease of the AUC value upon the application of MGTX. However, upon treatment with TRAM, CD8 cells showed a larger decrease in AUC and Max values than CD4 cells (Table 3).

In contrast with healthy subjects, no difference was detected between Th1 and Th2 or CD4 and CD8 cells in the sensitivity to IKCa1 channel inhibition among lymphocytes of RA patients. The difference in the decrease of AUC upon MGTX treatment was also smaller in RA than in healthy controls, however, the decrease was still more considerable in Th2 cells. Furthermore, in RA lymphocytes, MGTX inhibited CD8 cells to a higher extent instead of CD4 cells. These results indicate that MGTX, a specific blocker of the Kv1.3 channel acts differentially on calcium influx kinetics in major peripheral blood lymphocyte subsets of RA patients: Th2 and CD8 cells are inhibited more dominantly than Th1 and CD4 cells (Table 3).

\section{Kv1.3 channel expression in RA patients and healthy subjects}

The above mentioned results may be due to altered functionality or changes in the expression of Kv1.3 channels. Therefore, we measured the expression of these channels on the investigated lymphocyte subsets using a specific antibody. However, changes to the sensitivity of Kv1.3 channel inhibition seem to be unrelated to its altered expression, and therefore, do not explain our findings. Hence, functional alterations of the Kv1.3 channel must also play a role in differential sensitivity upon inhibition detected between RA patients and healthy controls. Of note, due to the lack of commercially available antibodies against the other investigated potassium channel, we could not perform a similar measurement in case of IKCa1.

\section{The specificity of Kv1.3 channel inhibition in RA patients}

Based on our results, the immunomodulatory effect of Kv1.3 channel inhibition is predominantly seen in cytotoxic (CD8) T cells in RA (Fig. 3). However, this effect does not seem to be as specific as reported before by Beeton and colleagues in case of TEM cells (Beeton et al. 2006), since anti-inflammatory Th2 cells are also affected to a noteworthy extent. This subset has an important role in counterbalancing the ongoing inflammatory process, and therefore 
its inhibition is not useful in the treatment of RA. A reason for limited specificity of Kv1.3 inhibition in peripheral lymphocytes might be the differential distribution of disease-associated autoreactive $T$ cells in RA patients on local and systemic levels. In the synovial fluid (locally), autoreactive TEM cells, expressing high numbers of Kv1.3 channels are abundantly present. However, this Kv1.3 pattern was not detected in peripheral blood T cells, because autoreactive TEM cells are infrequent in the circulation. Peripheral blood T cells were predominantly found to be naive and TCM cells (Beeton et al. 2006).

\section{Conclusion}

In conclusion, specific inhibition of the Kv1.3 channel acts differentially on calcium influx kinetics in major peripheral blood lymphocyte subsets of RA patients. Th2 and in particular CD8 cells are inhibited more dominantly than Th1 and CD4 cells. Changes to the sensitivity of Kv1.3 channel inhibition seem to be unrelated to its altered expression in the investigated lymphocyte subsets. The inhibitory effect does not seem to be as specific as reported before in case of autoreactive T cells, since anti-inflammatory Th2 cells are also affected to a noteworthy extent. A reason for limited specificity of Kv1.3 inhibition in peripheral lymphocytes might be the differential distribution of disease-associated autoreactive T cells in RA patients in the synovial fluid and the circulation.

\section{Acknowledgements}

This work was supported by the Hungarian Scientific Research Fund [OTKA; grant number 76316]; the Medical Research Council [grant number ETT 05-180/2009]; and a research grant from Roche Hungary Ltd.

\section{References}

Aletaha, D., Neogi, T., Silman, A.J., Funovits, J., Felson, D.T., Bingham 3rd, C.O., Birnbaum, N.S., Burmester, G.R., Bykerk, V.P., Cohen, M.D., Combe, B., Costenbader, K.H., Dougados, M., Emery, P., Ferraccioli, G., Hazes, J.M., Hobbs, K., Huizinga, T.W., Kavanaugh, A., Kay, J., Kvien, T.K., Laing, T., Mease, P., Ménard, H.A. Moreland, L.W., Naden, R.L., Pincus, T., Smolen, J.S., Stanislawska-Biernat, E., Symmons, D., Tak, P.P., Upchurch, K.S., Vencovský, J., Wolfe, F., Hawker, G., 2010. Rheumatoid arthritis classification criteria: an American College of Rheumatology/European League Against Rheumatism collaborative initiative. Arthritis Rheum. 62, 2569-2581.

Beeton, C., Wulff, H., Barbaria, J., Clot-Faybesse, O., Pennington, M., Bernard, D., Cahalan, M.D., Chandy, K.G., Béraud, E., 2001. Selective blockade of T lymphocyte K(+) channels ameliorates experimental autoimmune encephalomyelitis, a model for multiple sclerosis. Proc. Natl. Acad. Sci. U.S.A. 98, 13942-13947.

Beeton, C., Wulff, H., Standifer, N.E., Azam, P., Mullen, K.M., Pennington, M.W Kolski-Andreaco, A., Wei, E., Grino, A., Counts, D.R., Wang, P.H., LeeHealey, C.J., S Andrews, B., Sankaranarayanan, A., Homerick, D., Roeck, W.W., Tehranzadeh,
J., Stanhope, K.L., Zimin, P., Havel, P.J., Griffey, S., Knaus, H.G., Nepom, G.T., Gutman, G.A., Calabresi, P.A., Chandy, K.G., 2006. Kv1.3 channels are a therapeutic target for T cell-mediated autoimmune diseases. Proc. Natl. Acad. Sci. U.S.A. 103, 17414-17419.

Bucht, A., Larsson, P., Weisbrot, L., Thorne, C., Pisa, P., Smedegård, G., Keystone, E.C., Grönberg, A., 1996. Expression of interferon-gamma (IFN-gamma), IL-10, IL-12 and transforming growth factor-beta (TGFbeta) mRNA in synovial fluid cells from patients in the early and late phases of rheumatoid arthritis (RA). Clin. Exp. Immunol. 103, 357-367.

Buntinx, M., Ameloot, M., Steels, P., Janssen, P., Medaer, R., Geusens, P., Raus, J., Stinissen, P., 2002. Interferon-gamma-induced calcium influx in T lymphocytes of multiple sclerosis and rheumatoid arthritis patients: a complementary mechanism for T cell activation? J. Neuroimmunol. 124, 70-82.

Dolhain, R., ter Haar, N.T., Hoefakker, S., Tak, P.P., de Ley, M., Claassen, E., Breedveld, F.C., Miltenburg, A.M., 1996. Increased expression of interferon (IFN)-gamma together with IFN-gamma receptor in the rheumatoid synovial membrane compared with synovium of patients with osteoarthritis. Br. J. Rheumatol. 35, 24-32.

Dolmetsch, R.E., Xu, K., Lewis, R.S., 1998. Calcium oscillations increase the efficiency and specificity of gene expression. Nature 392, 933-936.

Fanger, C.M., Neben, A.L., Cahalan, M.D., 2000. Differential $\mathrm{Ca}^{2+}$ influx, KCa channel activity, and $\mathrm{Ca}^{2+}$ clearance distinguish Th1 and Th2 lymphocytes. J. Immunol. 164, 1153-1160.

Hu, L., Pennington, M., Jiang, Q., Whartenby, K.A., Calabresi, P.A., 2007. Characterization of the functional properties of the voltage-gated potassium channel Kv1. 3 in human CD4+ T lymphocytes. J. Immunol. 179, 4563-4570.

Kaposi, A.S., Veress, G., Vásárhelyi, B., Macardle, P., Bailey, S., Tulassay, T., Treszl, A., 2008. Cytometry-acquired calcium-flux data analysis in activated lymphocytes. Cytometry A 73, 246-253.

Nagy, G., Clark, J.M., Buzas, E., Gorman, C., Pasztoi, M., Koncz, A., Falus, A., Cope A.P., 2008. Nitric oxide production of T lymphocytes is increased in rheumatoid arthritis. Immunol. Lett. 118, 55-58.

Panyi, G., 2005. Biophysical and pharmacological aspects of $\mathrm{K}+$ channels in $\mathrm{T}$ lymphocytes. Eur. Biophys. J. 34, 515-529.

Panyi, G., Possani, L.D., Rodríguez de la Vega, R.C., Gáspár, R., Varga, Z., 2006. K+ channel blockers: novel tools to inhibit $\mathrm{T}$ cell activation leading to specific immunosuppression. Curr. Pharm. Des. 18, 2199-2220.

Szalay, B., Mészáros, G., Cseh, Á., Ács, L., Deák, M., Kovács, L., Vásárhelyi, B., Balog, A. 2012. Adaptive immunity in ankylosing spondylitis: phenotype and functional alterations of T-cells before and during infliximab therapy. Clin. Dev. Immunol., 808724.

Toldi, G., Folyovich, A., Simon, Z., Zsiga, K., Kaposi, A., Mészáros, G., Tulassay, T. Vásárhelyi, B., 2011. Lymphocyte calcium influx kinetics in multiple sclerosis treated without or with interferon beta. J. Neuroimmunol. 237, 80-86.

Toldi, G., Kaposi, A., Zsembery, Á., Treszl, A., Tulassay, T., Vásárhelyi, B., 2012. Human Th1 and Th2 lymphocytes are distinguished by calcium flux regulation during the first ten minutes of lymphocyte activation. Immunobiology 217, 37-43.

van der Graaff, W.L., Prins, A.P., Niers, T.M., Dijkmans, B.A., van Lier, R.A., 1999 Quantitation of interferon gamma- and interleukin-4-producing T cells in synovial fluid and peripheral blood of arthritis patients. Rheumatology (Oxford) 38, 214-220.

Verheugen, J.A., Vijverberg, H.P., 1995. Intracellular $\mathrm{Ca}^{2+}$ oscillations and membrane potential fluctuations in intact human $\mathrm{T}$ lymphocytes: role of $\mathrm{K}+$ channels in $\mathrm{Ca}^{2+}$ signaling. Cell Calcium 17, 287-300.

Wulff, H., Calabresi, P.A., Allie, R., Yun, S., Pennington, M., Beeton, C., Chandy, K.G. 2003. The voltage-gated Kv1.3 K+ channel in effector memory T cells as new target for MS. J. Clin. Invest. 111, 1703-1713.

Yang, P.T., Kasai, H., Zhao, L.J., Xiao, W.G., Tanabe, F., Ito, M., 2004. Increased CCR4 expression on circulating CD4+ T cells in ankylosing spondylitis, rheumatoid arthritis and systemic lupus erythematosus. Clin. Exp. Immunol. 138 342-347. 